Десницкая Валентина Николаевна

канд. физ.-мат. наук, доцент

ФГБОУ ВО «Санкт-Петербургский государственный экономический университет» г. Санкт-Петербург

DOI $10.31483 / r-21571$

\title{
МОДИФИКАЦИЯ МЕТОДА DЕА В ЗАДАЧЕ МНОГОКРИТЕРИАЛЬНОЙ ОПТИМИЗАЦИИ
}

Аннотация: в настоящее время существует больщое разнообразие подходов к решению задач многокритериальной оптимизацฺии, основанных на различных принциипах оптимальности. В работе сформулированы свойства, которыми представляется разумным наделить принципь оптимальности. $B$ статье также доказано, что широко распространенный метод многокритериальной оптимизации Data Envelopment Analysis (DEA) основан на принциие оптимальности, обладающцем этими свойствами. Это, безусловно, относится к достоинствам метода DEA. Метод DEA обладает также рядом недостатков. Предложенная в работе модификация метода позволяет избавиться от одного их них, «жертвуя» одним из свойств принциипов оптимальности. Исследование производится математическими методами. Все результаты математически доказаны.

Ключевые слова: многокритериальная оптимизащчия, принции оптимальности, метод DEA, распорядительный цүентр, относительная эфффективность, исправленная эффективность, принятие решений.

В основе оценки эффективности деятельности любой организации лежит, как правило, сопоставление результатов ее деятельности с затратами, обеспечивающими эти результаты. Особый интерес представляет случай, когда требуется дать сравнительную оценку эффективности деятельности каждой из нескольких однородных организаций. Под однородными организациями мы будем понимать 
организации, располагающие одинаковыми наборами ресурсов и предоставляющие одинаковые наборы результатов.

Существуют различные подходы к построению такого рода оценок. Ряд из них основан на использовании критериев, внешних по отношению к данной совокупности организаций.

Так, например, можно строить оценки эффективности деятельности организации, сравнивая суммарную стоимость затрат с суммарной стоимостью результатов. Однако такой подход применим только для случая, когда затраты и результаты можно оценить при помощи некоторой системы цен, что не всегда возможно. Например, при оценивании результатов работы больницы следует учесть такие ее разнородные характеристики, как количество койко-мест, наличие современного диагностического и лечебного оборудования, квалификация медицинского персонала, количество полностью вылеченных больных, количество больных, выписанных со значительным улучшением, выручка от предоставления коммерческих медицинских услуг и т. п. Не все из этих показателей представляется возможным оценить при помощи цен.

При построении внешнего критерия можно использовать экспертные оценки. Однако такой подход в большой степени субъективен.

Наша задача состоит в том, что оценить эффективность деятельности каждой организации из некоторой совокупности, не используя характеристик, внешних по отношению к данной совокупности.

Для этого поставим задачу в математической форме. Рассмотрим совокупность из $n$ однородных организаций. Будем называть эти организации распорядительными цุентрами (РЦ). Будем предполагать, что каждый РЦ имеет относительную самостоятельность в принятии решений. Деятельность каждого РЦ можно рассматривать как преобразование ресурсов в продукты, причем наборы ресурсов и продуктов для всех РЦ из данной совокупности одинаковы. Предположим, что все РЦ затрачивают один вид ресурса и производят $m$ видов продуктов. Таким образом, каждый РЦ можно описать вектором $X=\left(x_{1}, x_{2}, \ldots, x_{m}, x_{m+1}\right)$, где $x_{i}$-количество выпущенного продукта $i, i=1,2, \ldots, m$, a $x_{m+1}$-затраты 
peсурса. Введем естественное предположение: $x_{i} \geq 0$ при $i=1,2, \ldots, m$, а также будем считать, что $x_{m+1}>0$. Тогда деятельность каждого РЦ можно описать вектором $Y=\left(y_{1}, y_{2}, \ldots, y_{m}\right)$, где $y_{i}=\frac{x_{i}}{x_{m+1}}-$ выпуск продукта $i$, приходящийся на 1 единицу затрат ресурса. Вектор $Y=\left(y_{1}, y_{2}, \ldots, y_{m}\right)$ принадлежит $R_{+}^{m}-$ множеству векторов с неотрицательными компонентами из пространства $R^{m}$.

Рассмотрим совокупность $n$ распорядительных центров (РЦ). Деятельность РЦ с номером $j_{0}$ описывается вектором $Y^{j_{0}}=\left(y_{1}^{j_{0}}, y_{2}^{j_{0}}, \ldots, y_{m}^{j_{0}}\right)$, где $y_{i}^{j_{0}} \geq 0$, $i=1,2, \ldots, m$. Наша задача состоит в том, чтобы приписать вектору $Y^{j_{0}}$ некоторое число $\mu^{j_{0}}$, которым мы будем оценивать эффективность деятельности данного РЦ. При этом величина $\mu^{j_{0}}$ должна зависеть не только от вектора $Y^{j_{0}}$, но и от остальных векторов $Y^{j}=\left(y_{1}^{j}, y_{2}^{j}, \ldots, y_{m}^{j}\right), j=1,2, \ldots, n$ из данной совокупности. Таким образом, величина $\mu^{j_{0}}$ должна оценивать относительную эфффективность данного РЦ в рассматриваемой совокупности распорядительных центров. Иными словами, наша задача состоит в построении функции, определенной на множестве $C^{n}=\prod_{i=1, \ldots, n} R_{+}^{m}-$ n-кратном декартовом произведении множества $R_{+}^{m}$ на себя, сопоставляющей данному РЦ число, определяющее его эффективность в рассматриваемой совокупности.

Обозначим эту функцию $\gamma^{j_{0}}$. Будем считать, что значения этой функции принадлежат отрезку $[0 ; 1]$.

Сформулируем свойства, которым должна удовлетворять функция $\gamma^{j_{0}}$ в соответствии с нашими интуитивными представлениями об эффективности.

1. Независимость от масштаба.

Величина эффективности не должна зависеть от того, в каких единицах измеряется тот или иной показатель деятельности РЦ. Сформулируем это свойство математически. 
Пусть $Y^{j}=\left(y_{1}^{j}, y_{2}^{j}, \ldots, y_{m}^{j}\right), \tilde{Y}^{j}=\left(\lambda_{1} y_{1}^{j}, \lambda_{2} y_{2}^{j}, \ldots, \lambda_{m} y_{m}^{j}\right)$, где $\lambda_{i}>0, i=1,2, \ldots, m$. Тогда $\gamma^{j_{0}}\left(Y^{1}, Y^{2}, \ldots, Y^{n}\right)=\gamma^{j_{0}}\left(\tilde{Y}^{1}, \tilde{Y}^{2}, \ldots, \tilde{Y}^{n}\right)$.

2. Монотонность.

Будем считать, что увеличение любого из показателей деятельности РЦ увеличивает эффективность данного РЦ, при условии, что показатели деятельности остальных РЦ остаются неизменными.

3. Непрерывность.

Функция $\gamma^{j_{0}}$ непрерывна по каждому аргументу. Свойство непрерывности можно интерпретировать следующим образом: достаточно малое изменение любого из аргументов функции $\gamma^{j_{0}}$ влечет малое изменение значения этой функции.

В основу построения функции $\gamma^{j_{0}}$, удовлетворяющей свойствам $1-3$, можно взять метод оценки эффективности DEA (Data Envelopment Analysis).

Метод DEA - это относительно недавно появившийся метод оценки деятельности распорядительных центров из некоторой совокупности. Метод DEA был разработан в 1978 году и в ближайшие же годы получил широкое распространение. Приведем описание этого метода. Для этого введем ряд определений.

Определение 1 . Пусть $Y^{1}, Y^{2}, \ldots, Y^{n}$ - векторы из пространства $R^{m}$. Bыпуклой оболочкой этих векторов называется множество $C\left(Y^{1}, Y^{2}, \ldots, Y^{n}\right)=\left\{Y \in R^{m}: Y=\sum_{j=1}^{n} u_{j} Y^{j} ; \sum_{j=1}^{n} u_{j}=1 ; u_{j} \geq 0, j=1, \ldots n\right\}$.

Выпуклая оболочка конечного числа векторов является выпуклым многогранником в пространстве $R^{m}$. При этом вектор $Y^{j_{0}}$ называется вершиной множества $C\left(Y^{1}, Y^{2}, \ldots, Y^{n}\right)$, если $Y^{j_{0}} \in R^{m}$ и единственное представление вектора $Y^{j_{0}}$ в виде линейной комбинации векторов из совокупности $Y^{1}, Y^{2}, \ldots, Y^{n}$ имеет вид: $Y^{j_{0}}=\sum_{j=1}^{n} u_{j} Y^{j}$, при этом $u_{j_{0}}=1, u_{j}=0$ для $j \neq j_{0}$. 
Определение 2. Множество $D$ нормально относительно $R_{+}^{m}$, если справедливо равенство $\left(D-R_{+}^{m}\right) \bigcap R_{+}^{m}=D$.

Здесь под разностью двух множеств мы понимаем соответствующую операцию Минковского, а именно: $A-B=\{c \mid c=a-b, a \in A, b \in B\}$. Иными словами, если множество $A \subset R_{+}^{m}$, то $A$ нормально относительно $R_{+}^{m}$, если из того, что $a \in A$ следует, что $\lambda a \in A$, при $\lambda \in[0 ; 1]$.

Рассмотрим систему, состоящую из $n$ распорядительных центров. Каждый РЦ этой системы можно описать вектором $Y^{j}, j=1,2, \ldots, n$. Пусть $D$ - выпуклая, нормальная относительно $R_{+}^{m}$ оболочка совокупности векторов $\left\{Y^{1}, Y^{2}, \ldots, Y^{n}\right\}$. Поставим в соответствие каждому вектору $Y^{j}$ из данной совокупности такой вектор $Y_{p}^{j}$, что $Y^{j}=\mu^{j} Y_{p}^{j}$, где $0 \leq \mu^{j} \leq 1$ и вектор $Y_{p}^{j}$ лежит на границе множества $D$. В соответствии с терминологией, используемой в [4, с. 60], будем называть вектор $Y_{p}^{j}$ эталонным для вектора $Y^{j}$. Заметим, что в случае, когда вектор $Y^{j}$ лежит на границе множества $D$, он, очевидно, совпадает со своим эталонным вектором: $Y_{p}^{j}=Y^{j}$, в этом случае $\mu^{j}=1$.

Введем определение эффективности распорядительного центра в соответствии с методом DEA.

Определение. Рассмотрим распорядительный центр, деятельность которого описывается вектором $Y^{j_{0}}$. Эфффективностью данного РЦ будем называть величину $\mu^{j_{0}}$.

Поскольку для каждого вектора из совокупности $\left\{Y^{1}, Y^{2}, \ldots, Y^{n}\right\}$ эталонный вектор определяется множеством $D$, которое в свою очередь определяется всей совокупностью векторов $\left\{Y^{1}, Y^{2}, \ldots, Y^{n}\right\}$, эффективность каждого РЦ зависит от векторов совокупности $\left\{Y^{1}, Y^{2}, \ldots, Y^{n}\right\}$. Следовательно, введя понятие эффективности, мы тем самым построили функцию $\gamma^{j_{0}}: \gamma^{j_{0}}\left(Y^{1}, Y^{2}, \ldots, Y^{n}\right)=\mu^{j_{0}}$. 
Из определения следует, что эффективность распорядительных центров, описываемых векторами, лежащими на границе множества $D$, равна 1 , а в остальных случаях эффективность меньше 1.

Докажем, что построенная функция обладает требуемыми свойствами. Функция $\gamma^{j_{0}}$ определена на множестве $C^{n}$ и принимает значения из промежутка [0; 1]. Следующая теорема [5, с. 431] устанавливает важное свойство эффективности.

Теорема 1. Рассмотрим совокупность векторов $\left\{Y^{1}, Y^{2}, \ldots, Y^{n}\right\}$ из множества $R_{+}^{m}$. Эффективность распорядительного центра с номером $j_{0}$ является оптимумом целевой функции следующей задачи линейного программирования:

$$
\begin{aligned}
& F\left(u_{1}, u_{2}, \ldots, u_{n}\right)=u_{1}+u_{2}+\ldots+u_{n} \rightarrow \min \\
& Y^{1} u_{1}+Y^{2} u_{2}+\ldots+Y^{n} u_{n} \geq Y^{j_{0}} \\
& u_{j} \geq 0, j=1,2, \ldots, n
\end{aligned}
$$

Пользуясь этой теоремой, установим справедливость свойств 1-3.

1. Независимость от масштаба.

Задача (1) имеет $m$ ограничений. Умножим обе части каждого из ограничений на некоторые положительные числа $\lambda_{i}, i=1,2, \ldots, m$. Очевидно, полученная задача будет равносильна исходной. Следовательно, их оптимумы окажутся равными.

2. Монотонность.

Для установления этого свойства докажем ряд вспомогательных утверждений.

Лемма 1. Пусть $Y^{1}, Y^{2}, \ldots, Y^{n}$ - векторы из пространства $R^{m}$, а $C\left(Y^{1}, Y^{2}, \ldots, Y^{n}\right)$ выпуклая оболочка этих векторов. Пусть $Y^{n+1}$ - вектор из $R^{m}$, причем $Y^{n+1} \notin C\left(Y^{1}, Y^{2}, \ldots, Y^{n}\right)$. Тогда вектор $Y^{n+1}$ является вершиной множества $C\left(Y^{1}, Y^{2}, \ldots, Y^{n}, Y^{n+1}\right)$.

Доказательство. Предположим, что утверждение леммы неверно. Это значит, что существует такой набор чисел $u_{1}, \ldots, u_{n}, u_{n+1}$, удовлетворяющих условиям: 
$\sum_{j=1}^{n+1} u_{j}=1, u_{j} \geq 0, \quad$ что $\quad Y^{n+1}=\sum_{j=1}^{n+1} u_{j} Y^{j}, \quad$ и $\quad$ при этом $u_{n+1} \neq 1$. Тогда, очевидно, $\left(1-u_{n+1}\right) Y^{n+1}=\sum_{j=1}^{n} u_{j} Y^{j}$, причем $1-u_{n+1} \neq 0$. Следовательно, $Y^{n+1}=\sum_{j=1}^{n} \frac{u_{j}}{1-u_{n+1}} Y^{j}$. В этой сумме коэффициенты при векторах $Y^{1}, Y^{2}, \ldots, Y^{n}$ неотрицательны и в сумме составляют 1 . Значит, $Y^{n+1} \in C\left(Y^{1}, Y^{2}, \ldots, Y^{n}\right)$, что противоречит условию леммы.

Лемма 2.

Пусть $G_{1}$ и $G_{2}$ - две гиперплоскости в пространстве $R^{m}$, задаваемые уравнениями

$$
a_{1} x_{1}+\ldots+a_{m} x_{m}=b
$$

и

$$
a_{1} x_{1}+\ldots+a_{m} x_{m}=c
$$

соответственно, причем $b \neq 0, c \neq 0$. Пусть $l_{1}$ и $l_{2}$ - два луча, исходящих из начала координат $O$, задаваемых уравнениями в параметрической форме

$$
\left\{\begin{array} { l } 
{ x _ { 1 } = d _ { 1 } t } \\
{ \cdots \cdots } \\
{ x _ { m } = d _ { m } t }
\end{array} \text { и } \left\{\begin{array}{l}
x_{1}=k_{1} t \\
\cdots \cdots \\
x_{m}=k_{m} t
\end{array},\right.\right.
$$

при $t \geq 0$.

Пусть $A_{1}$ и $B_{1}$ - точки пересечения луча $l_{1}$ с гиперплоскостями $G_{1}$ и $G_{2}$ соответственно, а $A_{2}$ и $B_{2}$ - точки пересечения луча $l_{2}$ с гиперплоскостями $G_{1}$ и $G_{2}$ соответственно. Тогда

$$
\frac{\left\|O A_{1}\right\|}{\left\|O B_{1}\right\|}=\frac{\left\|O A_{2}\right\|}{\left\|O B_{2}\right\|} .
$$

Доказательство. Из уравнений гиперплоскостей $G_{1}$ и $G_{2}$ и из уравнений лучей $l_{1}$ и $l_{2}$ найдем координаты точек пересечения лучей с гиперплоскостями:

$$
A_{1}\left(\frac{d_{1} b}{\sum_{i=1}^{m} d_{i} a_{i}}, \ldots, \frac{d_{m} b}{\sum_{i=1}^{m} d_{i} a_{i}}\right), B_{1}\left(\frac{d_{1} c}{\sum_{i=1}^{m} d_{i} a_{i}}, \ldots, \frac{d_{m} c}{\sum_{i=1}^{m} d_{i} a_{i}}\right), A_{2}\left(\frac{k_{1} b}{\sum_{i=1}^{m} k_{i} a_{i}}, \ldots, \frac{k_{m} b}{\sum_{i=1}^{m} k_{i} a_{i}}\right), B_{2}\left(\frac{k_{1} c}{\sum_{i=1}^{m} k_{i} a_{i}}, \ldots, \frac{k_{m} c}{\sum_{i=1}^{m} k_{i} a_{i}}\right) .
$$


Тогда, как легко вычислить, $\frac{\left\|O A_{1}\right\|}{\left\|O B_{1}\right\|}=\frac{b}{c}$ и $\frac{\left\|O A_{2}\right\|}{\left\|O B_{2}\right\|}=\frac{b}{c}$, что и доказывает справедливость утверждения леммы.

Лемма 3. Рассмотрим в пространстве $R^{m}$ гиперплоскость $G$, не содержащую начало координат, и луч $l$, исходящий из начала координат $O$. Гиперплоскость $G$ разбивает $R^{m}$ на два полупространства: $R_{0}^{m}$ - полупространство, содержащее начало координат $O$, и $R_{1}^{m}$ - полупространство, не содержащее начало координат $O$. Пусть точка $A \in G \cap l$, точка $B \in R_{0}^{m} \cap l$, точка $C \in R_{1}^{m} \cap l$. Тогда $\|O B\| \leq\|O A\| \leq\|O C\|$.

Доказательство. Пусть гиперплоскость $G$ задается уравнением $a_{1} x_{1}+\ldots+a_{m} x_{m}=b$, а луч $l-$ системой уравнений $\left\{\begin{array}{l}x_{1}=d_{1} t \\ \cdots \ldots . . \\ x_{m}=d_{m} t\end{array}\right.$. Тогда в точке $A$ значение параметра $t_{A}=\frac{b}{\sum_{i=1}^{m} a_{i} d_{i}}$. Для точек на луче $l$, лежащих в одном из полупространств значение параметра $t: t \leq t_{A}$, а для точек на луче $l$, лежащих в другом полупространстве значение параметра $t: t \geq t_{A}$. Очевидно, что чем больше значение $t$ для точки на луче $l$, тем больше расстояние от этой точки до начала координат. Поскольку для начала координат $O$ значение параметра $t=0$, справедливо $\|O B\| \leq\|O A\|$, а следовательно, справедливо и $\|O A\| \leq\|O C\|$. Лемма доказана.

Следующая теорема устанавливает монотонность функции $\gamma^{j_{0}}\left(Y^{1}, Y^{2}, \ldots, Y^{n}\right)=\mu^{j_{0}}$

Теорема 2. Пусть $\left\{Y^{1}, Y^{2}, \ldots, Y^{n}\right\}$ - совокупность векторов из $R_{+}^{m}$. Рассмотрим вектор $\quad Y^{j_{0}} \in R_{+}^{m}, \quad$ удовлетворяющий $\quad$ условию: $\quad Y^{j_{0}} \geq Y^{j_{0}}$. Тогда $\gamma^{j_{0}}\left(Y^{1}, \ldots, Y^{j_{0}}, \ldots, Y^{n}\right) \geq \gamma^{j_{0}}\left(Y^{1}, \ldots, Y^{j_{0}}, \ldots, Y^{n}\right)$ 
Смысл теоремы состоит в том, что если один из РЦ улучшает свою деятельность хотя бы по одному из показателей, а при этом деятельность остальных РЦ не меняется, то эффективность данного РЦ не уменьшается.

Доказательство теоремы.

По определению эффективности $\gamma^{j_{0}}\left(Y^{1}, \ldots, Y^{j_{0}}, \ldots, Y^{n}\right) \leq 1$.

1. Рассмотрим сначала случай, когда вектор $Y^{j_{0}}$ не принадлежит множеству $D$. Тогда вектор $Y^{j_{0}}$ является вершиной выпуклой оболочки совокупности векторов $\left\{Y^{1}, \ldots, Y^{j_{0}}, \ldots, Y^{n}\right\}$, следовательно, по лемме $1, \gamma^{j_{0}}\left(Y^{1}, \ldots, Y^{j_{0}}, \ldots, Y^{n}\right)=1$, что доказывает утверждение теоремы для данного случая.

2. Рассмотрим теперь случай, когда вектор $Y^{j_{0}}$ принадлежит множеству $D$. В этом случае, очевидно, вектор $Y^{j_{0}}$ также является внутренней точкой множества $D$. Пусть $Y_{p}^{j_{0}}-$ эталонный вектор для вектора $Y^{j_{0}}$. Рассмотрим грань множества $D$, содержащую вектор $Y_{p}^{j_{0}}$, и пусть $G_{p}^{0}$ - гиперплоскость в пространстве $R^{m}$, содержащая эту грань.

Пусть $G^{0}$ - гиперплоскость в пространстве $R^{m}$, проходящая через точку $Y^{j_{0}}$, параллельная гиперплоскости $G_{p}^{0}$. Рассмотрим лучи $l^{0}$ и $\tilde{l}^{0}$, исходящие из начала координат $O$, и проходящие через точки $Y^{j_{0}}$ и $Y^{j_{0}}$ соответственно. Обозначим точки пересечения луча $\tilde{l}^{0}$ с плоскостями $G^{0}$ и $G_{p}^{0}$ буквами $A$ и $B$ соответственно. Тогда по лемме 2 справедливо равенство $\frac{\left\|Y^{j_{0}}\right\|}{\left\|Y_{p}^{j_{0}}\right\|}=\frac{\|O A\|}{\|O B\|}$.

Из выпуклости множества $D$ следует, что оно расположено по одну сторону от гиперплоскости $G_{p}^{0}$, а именно в полупространстве, содержащем начало координат $O$. Следовательно, по лемме 3 имеем неравенство $\left\|Y_{p}^{j_{0}}\right\| \leq\|O B\|$.

Множество, содержащее все возможные векторы $Y^{j_{0}}$ также, очевидно, выпукло. Значит оно расположено по одну сторону от гиперплоскости $G^{0}$, a именно 
в полупространстве, не содержащем начало координат $O$. Следовательно, по лемме 3 имеем неравенство $\left\|Y^{j_{0}}\right\| \geq\|O A\|$.

Таким образом, получаем неравенство: $\frac{\left\|Y^{j_{0}}\right\|}{\left\|Y_{p}^{j_{0}}\right\|} \leq \frac{\left\|Y^{j_{0}}\right\|}{\left\|Y_{p}^{j_{0}}\right\|}$. Учитывая, что выражение в левой части неравенства равно $\gamma^{j_{0}}\left(Y^{1}, \ldots, Y^{j_{0}}, \ldots, Y^{n}\right)$, а в правой части$\gamma^{j_{0}}\left(Y^{1}, \ldots, Y^{j_{0}}, \ldots, Y^{n}\right)$, получим, что утверждение теоремы верно.

3. Непрерывность.

Непрерывность функции $\gamma^{j_{0}}$ по каждому аргументу вытекает из непрерывности оптимального значения целевой функции по каждому параметру задачи линейного программирования.

Таким образом, мы установили, что метод DEA дает возможность оценить эффективность деятельности распорядительного центра при помощи функции, удовлетворяющей свойствам независимости от масштаба, монотонности и непрерывности.

Способ оценивания эффективности деятельности распорядительного центра, как правило, влияет на политику, проводимую ее руководством, а именно на принятие решений о распределении имеющихся ресурсов. Выбор политики формирует значение показателей деятельности РЦ. Метод DEA, как было отмечено выше, дает оценку эффективности каждого РЦ, зависящую не только от его собственной деятельности, но и от деятельности остальных распорядительных центров совокупности. Так, например, оценка эффективности РЦ может измениться в ту или иную сторону только за счет изменения показателей деятельности некоторых других РЦ из совокупности, а при этом значения показателей деятельности данного РЦ останутся неизменными.

Таким образом, оценивание методом DEA может привести к таким явлениям, как увеличение оценки эффективности деятельности РЦ за счет изменения соотношения между показателями. Так, например, существенное увеличение значения одного из показателей за счет уменьшения других, может привести к 10 https://phsreda.com

Содержимое доступно по лицензии Creative Commons Attribution 4.0 license (CC-BY 4.0) 
изменению множества $D$, и вывести вектор $Y$, соответствующий данному РЦ на границу $D$. В результате этого эффективность данного РЦ станет равной 1 , что не всегда представляется оправданным.

Рассмотрим модификацию метода DEA, позволяющую в какой-то мере сгладить этот недостаток метода.

Определение. Рассмотрим совокупность распорядительных центров, описываемую совокупностью векторов $\left\{Y^{1}, Y^{2}, \ldots, Y^{n}\right\}$. Вектор $\bar{Y}=\frac{1}{n} \sum_{j=1}^{n} Y^{j}$ называется средним вектором совокупности.

Пусть $\varphi^{j}$ - угол между векторами $Y^{j}$ и $\bar{Y}$. Введем в определение эффективности распорядительного центра поправочный множитель $\alpha^{j}=\cos \varphi^{j}$.

Определение. Исправленной эффективностью распорядительного центра, описываемого вектором $Y^{j}$, будем называть величину $\beta^{j}=\mu^{j} \cdot \alpha^{j}$.

Определенная таким образом величина $\beta^{j}$ оценивает не только степень близости вектора $Y^{j}$ от его эталонного значения $Y_{p}^{j}$, но и степень близости $Y^{j}$ от среднего вектора совокупности $\bar{Y}$, которая измеряется косинусом угла между этими векторами. Очевидно, что для каждого распорядительного центра с номером $j$ величина $\beta^{j}$ зависит от всех векторов совокупности $\left\{Y^{1}, Y^{2}, \ldots, Y^{n}\right\}$. Таким образом, $\beta^{j}$ является функцией от векторов $Y^{1}, \ldots, Y^{n}$.

Проверим, обладает ли функция $\beta^{j}$ свойствами независимости от масштаба, монотонности и непрерывности.

1. Независимость от масштаба.

Величина $\alpha^{j}=\cos \varphi^{j}$, очевидно, не зависит от единиц измерения компонент векторов $Y^{1}, \ldots, Y^{n}$, а для величины $\mu^{j}$ независимость от масштаба была доказана выше. Следовательно, и произведение $\alpha^{j}$ и $\mu^{j}$ не зависит от масштаба.

2. Монотонность.

Свойство монотонности было установлено для функции $\gamma^{j}$. Что касается свойства монотонности для функции $\beta^{j}$, то оно, вообще говоря, не выполняется. Это соответствует самой идее введения понятия исправленной эффективности. 
Роль поправочного коэффициента состоит именно в том, чтобы уменьшить оценку эффективности деятельности РЦ с номером $j$, в случае, когда большое значение этой оценки достигается существенным отклонением вектора $Y^{j}$ от $\bar{Y}$ среднего вектора совокупности $\left\{Y^{1}, Y^{2}, \ldots, Y^{n}\right\}$.

Следующий пример демонстрирует отсутствие монотонности для функции $\beta^{j}$.

Пример. Рассмотрим совокупность из 35 распорядительных центров, описываемых векторами $Y^{1}, \ldots, Y^{35}$. При этом $Y^{j}=(1,75 ; 6,5)$ для $j=1,2, \ldots, 31$, $Y^{32}=(1 ; 8), Y^{33}=(1 ; 6), Y^{34}=(3 ; 7), Y^{35}=(2 ; 5)$. Тогда, как нетрудно установить, средний вектор совокупности совпадает с вектором $Y^{1}: \bar{Y}=(1,75 ; 6,5)$. Вычислим эффективность РЦ, описываемого вектором $Y^{1}$, решая задачу линейного программирования:

$$
\begin{aligned}
& F\left(u_{1}, u_{2}, \ldots, u_{35}\right)=u_{1}+u_{2}+\ldots+u_{35} \rightarrow \min \\
& Y^{1} u_{1}+Y^{2} u_{2}+\ldots+Y^{35} u_{35} \geq Y^{1} \\
& u_{j} \geq 0, j=1,2, \ldots, 35
\end{aligned}
$$

Получим, что эффективность данного распорядительного центра $\mu_{1}=0,8675$. Поскольку в данном случае $Y^{1}=\bar{Y}$, угол между этими векторами равен 0 , и, следовательно, $\alpha_{1}=1$ и $\beta_{1}=\mu_{1}=0,8675$.

Предположим теперь, что вектор $Y^{1}$ заменили на вектор $Y^{1}=(7 ; 6,5)$, при этом $Y^{1} \geq Y^{1}$. Тогда эффективность данного РЦ станет равной $\mu_{1}=1$. Это легко установить графически. При этом средний вектор измененной совокупности станет равным $Y=(1,91 ; 6,31)$. Вычислим $\alpha_{1}$ - косинус угла между векторами $Y$ и $Y^{1}$ : $\alpha_{1}=0,8638$. Таким образом, исправленная эффективность данного РЦ окажется равной $\beta_{1}=\alpha_{1}=0,8638$. Следовательно, исправленная эффективность, вообще говоря, свойством монотонности не обладает. 


\section{3. Непрерывность.}

Исправленная эффективность обладает свойством непрерывности, так как является произведением двух функций, каждая из которых, очевидно, непрерывна.

Таким образом, предложенная модификация метода DEA дает возможность оценить не только близость вектора, описывающего деятельность распорядительного центра, к своему эталонному вектору, но и близость к среднему вектору совокупности распорядительных центров.

В заключение заметим, что задачи многокритериальной оптимизации служат, как правило, для поддержки принятия решений. Многообразие существующих методов приводит к тому, что принятые на основании их решения могут значительно отличаться друг от друга и приводить к далеким друг от друга, даже к противоположным, результатам. Поэтому представляется весьма важным сформулировать общие свойства, которыми мы хотели бы наделить понятие оптимального, и проверять каждый принцип оптимальности на наличие у него этих свойств. При этом отметим, что ни один подход не приведет к принятию безусловно «правильного» решения, выбор решения всегда остается за человеком.

\section{Список литературы}

1. Данциг Д. Линейное программирование, его применения и обобщения. М.: Прогресс, 1966.

2. Десницкая В.Н. Задачи многокритериальной оптимизации: метод DEA и выпуклый анализ // Современный взгляд на проблемы экономики и менеджмента: Сборник научных трудов по итогам международной научно-практической конференции. - Уфа: Инновационный центр развития образования и науки, 2014.

3. Рокафеллар. Р. Выпуклый анализ. - М.: Мир, 1973.

4. Федотов Ю.В. Метод DEA: анализ свертки данных // Российский журнал менеджмента. Т. 10. - 2012. - №2. С. 
5. Charnes A. Measuring the efficiency of decision-making units / A. Charnes, W.W. Cooper, E. Rhodes // European Journal of Operation Research. T. 2. - 1978. №6. 